\title{
Internal Structure Evaluation of Three-Dimensional Calcium Phosphate Bone Scaffolds: A Micro-Computed Tomographic Study
}

\author{
Leenaporn Jongpaiboonkit ${ }^{* \dagger}$ and John W. Halloran ${ }^{\star *}$ \\ Department of Materials Science and Engineering, University of Michigan, Ann Arbor, Michigan 48109
}

Scott J. Hollister

Department of Biomedical Engineering, Department of Mechanical Engineering, and Department of Surgery, University of Michigan, Ann Arbor, Michigan 48109

\begin{abstract}
Micro-computed tomography ( $\mu-\mathrm{CT})$ was used to examine the fidelity of three-dimensional (3D) bioceramic scaffolds made from dicalcium phosphate dihydrate (DCPD) cement. The scaffolds were produced with a multi-step indirect solid freeform fabrication method. Accuracy and fidelity were examined after each step. The pre-tracing of the perimeter of a layer in the mold fabrication step caused the round-off of the square channels from the design. However, the overall shape of the scaffold was not significantly different from the wax mold. There is relatively no change in the strut and the channel-size measurements between the computer-generated model and the molds fabricated by $3 D$ printing. The change in volume fraction of DCPD cement scaffolds is higher than biphasic scaffolds due to the internal pores. These results demonstrated that $\mu-C T$ is a very useful tool to analyze and evaluate the internal architectural of the complicated 3D scaffold-fabrication process.
\end{abstract}

\section{Introduction}

CAfFold external and internal architectures, including pore size, pore shape, pore distribution, and pore connection, play important roles in both in vivo and mechanical performance of the implants. Klein et al. ${ }^{1}$ showed that macroporosity plays a role in the rate of bone substitution in dog femora. Several methods for manufacturing porous calcium phosphate cement (CPC) with controlled pore channels have been developed. ${ }^{2-4}$ The ability to design and fabricate controlled scaffold architectures will make it possible to study rigorously how the scaffold affects bone regeneration. Scaffold architecture design can be readily accomplished using the image-based design (IBD) method, which was developed by Hollister et al. ${ }^{5,6}$ and Taboas et $a l^{7}$ to design and optimize scaffold architecture. The complicated designs created by IBD must be manufactured with solid freeform (SFF) fabrication to control scaffold external shape and internal pore interconnectivity and geometry. It is important to characterize the interior of these scaffolds to assure that the internal architecture accurately replicates the design with good fidelity. In this paper, we perform quantitative analysis of the interior of complex scaffolds using micro-computed tomography $(\mu-\mathrm{CT})$ to assess the fidelity of scaffolds at each stage of their fabrication. Indirect SFF (ISFF) has been developed by Chu et $a l^{4,8}$ The technique enables three-dimensional (3D)

T. Troczynski-contributing editor

Manuscript No. 21140. Received November 10, 2005; approved April 19, 2006

This work is supported in part by the National Institute of Health (NIH) Grant No. NIH DE 13608 .

${ }^{*}$ Member, American Ceramic Society.

**Fellow, American Ceramic Society.

†uthor to whom correspondence should be addressed. e-mail: ljongpai@umich.edu ceramic and polymer-ceramic scaffolds. ${ }^{7}$ Reilly et al. ${ }^{9}$ applied the ISFF method to manipulate $3 \mathrm{D}$ electromagnetic bandgap structures. ISFF fabrication has proven to be a suitable technique for the shaping of complex geometries and advanced design, which is not possible with conventional manufacturing methods. Although these complex 3D structures were precisely measured for the externally visible structures and characterized physically, the internal structures were not characterized and how well the fabricated internal architecture replicates the design has not been rigorously quantified.

Previously, we reported ISFF fabrication for bioceramic bone-tissue engineering scaffolds ${ }^{10,11}$ made from dicalcium phosphate dihydrate (DCPD) cement. CPC is gaining increasing popularity for bone repair and bone substitution, not only for its excellent biocompatibility and bone-replacement behavior over long periods ${ }^{12,13}$ but also for its similar osteoconductivity to autologous bone. The first self-setting cement formulations consisting of calcium phosphates were introduced in 1983 by Brown and Chow. ${ }^{14}$ In this present work, we studied DCPD cement based on $\beta$-tricalcium phosphate $(\beta$-TCP)-calcium phosphate monobasic, monohydrate (MCPM) in an aqueous system. ${ }^{15}$ This cement sets at physiological temperatures, and can be simply injected into the bone defects; the formulations to be molded can also be adjusted during the operation. Here, we use DCPD cement to mold scaffolds with a specific design ${ }^{16}$ of voids and struts. Unlike the fluid ceramic slurries in previous work, ${ }^{8,9}$ the DCPD was a more viscous paste before setting. The complex 3D CPC scaffolds fabricated by the ISFF method were thoroughly examined using a nondestructive technique before in vivo experiments. $^{11}$ 3D $\mu$-CT analysis allows us to collect data from the same specimens to observe and evaluate the complicated scaffold-fabrication process in order to assess the fidelity of the fabrication technique.

\section{Experimental Procedure}

A schematic of scaffold fabrication methods is outlined in Fig. 1. Five steps are involved: (1) design, leading to a CAD model; (2) building of the "lost mold" from wax and polysulfonamide (PSA) with solid ink-jet printing; (3) dissolution of the wax to leave the PSA lost mold; (4) casting of the cement suspension in the PSA mold; and (5) dissolution of the PSA mold. We use $\mu$-CT to examine the fidelity of each step.

\section{(1) Materials}

MCPM 99\% (Strem Chemicals, Newburyport, MA), and $\beta$ TCP (particle size was around $30 \mu \mathrm{m}$, Plasma Biotel Ltd., North Derbyshire, UK) were used as received. The scaffold molds were manufactured from PSA (or Protobuild ${ }^{\mathrm{TM}}, T_{\mathrm{m}}=85^{\circ}-107^{\circ} \mathrm{C}$ ) and wax (Protosupport ${ }^{\mathrm{TM}}, T_{\mathrm{m}}=60^{\circ}-75^{\circ} \mathrm{C}$ ), supplied by SolidScape (SolidScape Inc., Merrimack, NH). Bioact (Petroferm Inc., 


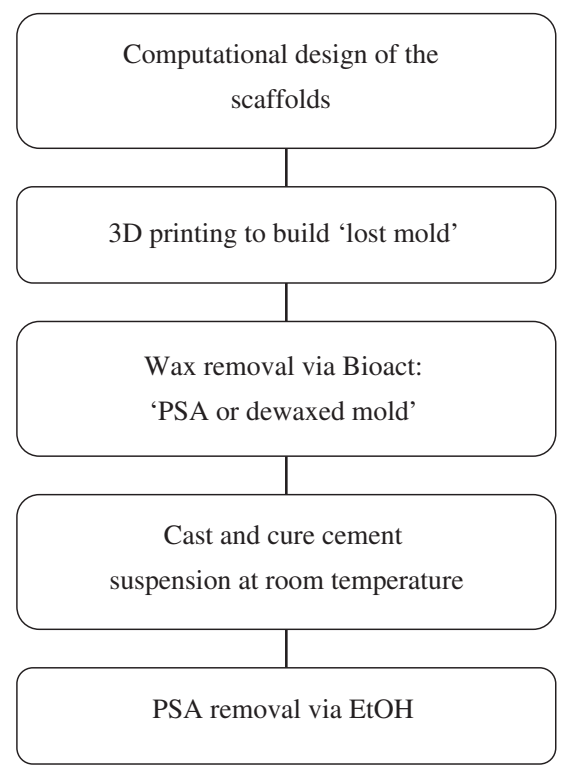

Fig. 1. Schematic chart of the indirect solid freeform procedure.

Fernanadina Beach, FL) was used to remove the wax. Ethanol (99\% EtOH, Sigma-Aldrich, St. Louis, MO) was used to remove the PSA.

\section{(2) Computational Design and Scaffold Fabrication}

Cylindrical porous scaffolds with 3D orthogonal periodic architectures were designed using the IBD method developed by Hollister et al. ${ }^{6}$ and Lin et al. ${ }^{17}$ This technique not only allows the creation of complex internal pore architectures but also allows the scaffold external shape to match implant site requirements using specific image data. The scaffolds were cylindrical with $8.85 \mathrm{~mm}$ diameter and $6.05 \mathrm{~mm}$ height, with square channels (channel size $=650 \mu \mathrm{m}$ ) separated at equal distances by struts (strut size $=550 \mu \mathrm{m}$ ). This defined orthogonally branching and interconnected pore channels with a solid volume fraction of the design $=42 \%$. Note that the solid volume fraction refers to the fraction of the entire designed scaffold volume that is occupied by calcium phosphate bioceramic materials. Figure 2 presents five consecutive steps from design to finished scaffold: (1) design model, (2) as-built wax molds (wax+PSA) of the scaffold, (3) dewaxed molds - the PSA lost mold determines the void channels in the scaffold, (4) cast molds - with DCPD cement cast around and inside the PSA lost mold, and (5) scaffold - after removal of PSA, a replica of the model in set cement.

The scaffold mold design files (Step 1-design model) were imported into Modelworks software (SolidScape Inc.) for multiple slicing to support a single build, and converted into input files for 3D printing. Multiple copies of the wax mold (Step 2wax mold) were produced using the ModelMaker II rapid prototyping system (SolidScape Inc.), which constructs models by sequentially depositing two different materials - molten red wax, followed by molten green PSA - to make one layer of the mold. The red wax is deposited to define the areas that will eventually be calcium phosphate. The green PSA is deposited to fill the area, which will eventually be pore channels. A horizontal rotary cutter is used to smooth the top surface, to control the layer thickness, $0.0508 \mathrm{~mm}$, for this study. The process was repeated layer by layer until the entire wax mold was constructed. Dewaxed molds were obtained by melting the red wax and removing residual wax in warm Bioact, leaving the green PSA, which determines the dewaxed mold (Step 3-dewaxed mold). A suspension was formed from a 1:1 molar ratio of MCPM and $\beta$-TCP powder with varying amounts of distilled water added. The viscosity of the cement is $\mathrm{F} 100 \mathrm{mPa} \cdot \mathrm{s}$ at $\mathrm{L} / \mathrm{P}$ (liquid-to-powder) ratio $=1.00$. The slip was cast in the dewaxed molds (Step 4 -cast mold) and cured at room temperature $\left(23^{\circ} \mathrm{C}\right)$. The cement set within $15 \mathrm{~min}$. The PSA molds were removed by washing in $70 \% \mathrm{EtOH}$ and drying in air, resulting in the finished scaffold (Step 5-scaffold).

\section{(3) Characterization}

The interior architectures of the wax molds (Step 2), dewaxed molds (Step 3), cast molds (Step 4), and scaffolds (Step 5) were conducted by using an MS-130 high-resolution $\mu$-CT scanner (GE Medical Systems, Toronto, Canada) with a $\mathrm{Cu}$ filter at $15 \mu \mathrm{m}$ voxel resolution, using a shutter speed of $3000 \mathrm{~ms}$, with a $2 \times 2$ bin size, at $100 \mathrm{kV}$ and $110 \mathrm{~mA}$. Each specimen $(n=6)$ was sequentially scanned in air. For each sample, a total of 400 micro-tomographic slices were acquired. Measurements were stored in 3D image arrays with an isotropic voxel size of $15 \mu \mathrm{m}$ using GEMS MicroView software (GE Medical Systems). The reconstructed $3 \mathrm{D}$ data sets were used to measure the pore size and wall size in the $x, y$, and $z$ directions.

The volume fraction of solid was calculated by defining a region of interest that covered the entire mold/scaffold, and then using a thresholding procedure with GEMS MicroView software. There is a distinct density difference between the mold and air, or cement and mold, or cement and air. The density histogram had a sharp cut-off, so an unambiguous threshold could be attained.

\section{Results and Discussion}

Each specimen was scanned in air using $\mu$-CT before proceeding to the subsequent step. The measurements were performed randomly on each $2 \mathrm{D}$ image slide for at least 10 image slides per specimen in all directions $(x, y$, and $z$ ). Figure 3 illustrates the slides of reconstructed $\mu$-CT images for the interior measurements. Figure 3(a) shows a 2D reconstructed image of the wax mold (Step 2). The light gray areas indicate PSA, which will become the pore channels, and the darker contrast represents wax, which will become the scaffold struts. Note the dark contrast of voids where the wax was not deposited around and between the square channels. These voids do not play a role because the PSA is removed.

The theoretical values for the scaffold dimensions were calculated from the computer model used to design the molds. The values of physical measurements (interior channel size and strut size) of wax molds, dewaxed molds, and cement scaffolds are summarized in Table I. The wax mold (Step 2) is an accurate reproduction of the CAD model to within $\pm 10 \mu \mathrm{m}$ of both the struts (red wax) and the channels (green PSA). The removal of the wax in Step 3 appears to decrease both the channels and struts, which could be due to the poor contrast between air (as the wax was removed) and PSA. However, Student's $t$-test was performed and showed statistically no significance. In Step 4 (cast mold), the measured size of the cement struts is the same as previous air in Step 3 and statistically the same as the wax mold and the CAD design. The measured channel size of the PSA is relatively close to the CAD design. After the PSA removal with $70 \%$ EtOH in Step 5, the final scaffold strut size appeared to be smaller than it was in the cast mold and also smaller than that of the design by $50 \mu \mathrm{m}$ (or $10 \%$ ), while the channel size was larger than the design by $40 \mu \mathrm{m}$ (or $6 \%$ ). The reduction in strut size in the final step is not clear, although we did not investigate any chemical reaction of the cement with PSA solution in EtOH. In contrast, the PSA dissolution in acetone did react with the porous cement scaffolds, causing the collapse of the scaffold. However, we conclude that there is relatively no change in the channel size from the computer-generated model to the molds physically fabricated by $3 \mathrm{D}$ printing. Results demonstrate that ModelMaker II is capable of accurately creating wax molds with a channel size of $650 \mu \mathrm{m}$, and a strut size of $550 \mu \mathrm{m}$ corresponding to the designed dimensions (CAD design). It has been shown that the smallest feature that the ModelMaker II can produce is $181 \pm 8 \mu \mathrm{m} .^{18}$ 


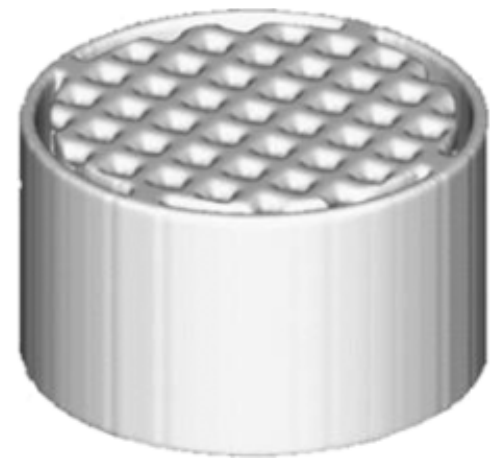

(a)

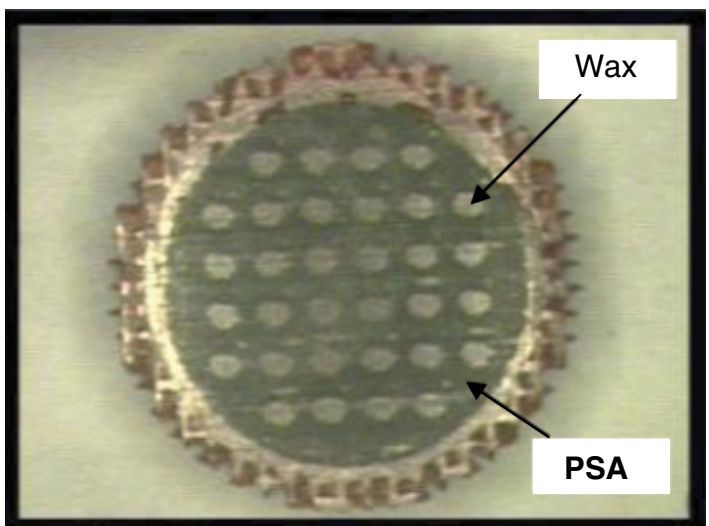

(b)

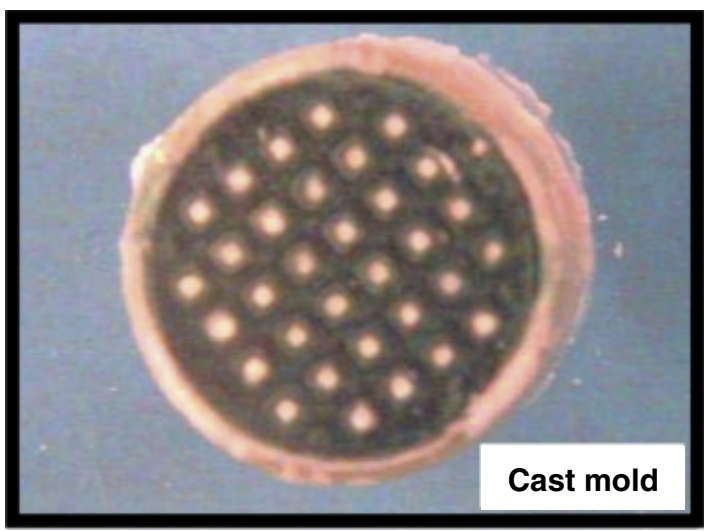

(d)

\section{Orthogonal design :}

$$
\begin{aligned}
& \text { Channel size (pore) }=650 \mu \mathrm{m} \\
& \text { Wall size (strut) }=550 \mu \mathrm{m} \\
& \text { Total macroporosity }=50 \%
\end{aligned}
$$

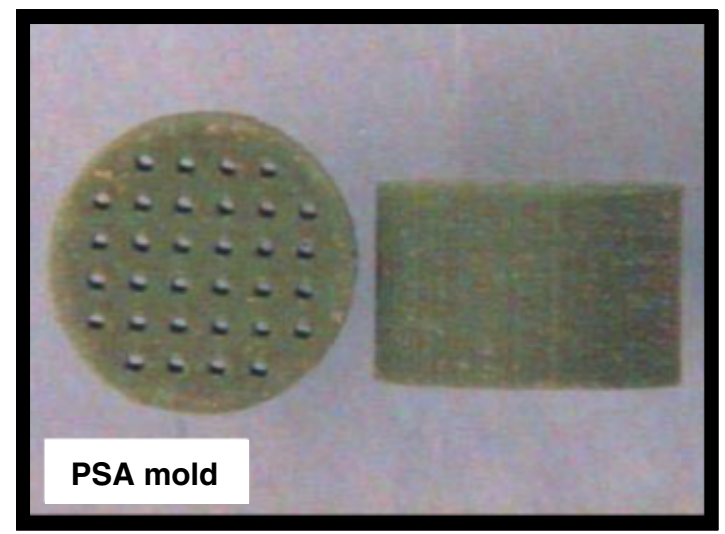

(c)

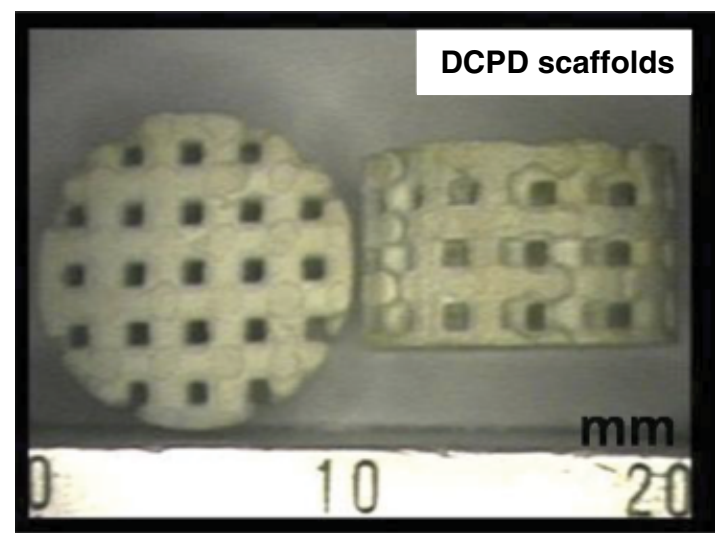

(e)

Fig. 2. Scaffold fabrication by indirect solid freeform: (a) Step 1 - cylindrical design mold with $650 \mu \mathrm{m}$ square channels and $550 \mu \mathrm{m}$ struts size, (b) Step 2-wax mold fabricated by 3D printing, (c) Step 3-polysulphonamide (PSA) or dewaxed mold, (d) Step 4 - cast mold, and (e) Step 5- dicalcium phosphate dihydrate (DCPD) scaffold after PSA removal.

To examine the change in volume fraction of solid, the volume of interest was selected within the reconstructed $3 \mathrm{D}$ image from the $\mu-C T$ scanner and a voxel rendering was performed for volume-fraction calculations. Figure 4 shows the image registration of the computer-generated design to the reconstructed CT image of the wax mold using SolidView/Pro 2003.1 (Solid Concepts Inc.). Figure 4(a) shows the offset from the center of the wax mold and the design, and the round-off of the square channels of the fabricated mold. In a rapid prototype using ModelMaker II, the smooth surface quality can be achieved by pre-tracing the perimeter of a layer prior to filling in the interior, which caused the round-off of the square channel from the design as seen in Fig. 4(a). The conversion of the CAD model to the STL (stereolithography) format could cause the mismatch of the fabricated mold from the design. The STL format (universal RP build format) comprised of a 3D surface as an assembly of planar triangles and normal vectors (perpendicular to the surface of the facet, pointing toward the outside part); thus, they cannot represent curved surfaces exactly. Increasing the number of triangles improves the approximation, but at the cost of a larger file size. Therefore, the accuracy and manageability need to be balanced. After the mold-making process, the reconstructed $2 \mathrm{D}$ image of $\mathrm{DCPD}$ scaffold was overlaid on the image of the wax mold on the $x y$ plane and was almost perfectly matched. The struts of the DCPD scaffold were smaller than the design and were rounded off similar to the finished mold (Fig. 4(b)). In this study, the size of a micro-droplet from the spray is $0.076 \mathrm{~mm}$ (from the manufacturer's specifications) by default.

Figure 5 shows the comparison of the relative volume change of the solid at each step of the fabrication. There is no change in volume of the computer model; thus, the relative volume change of the mold design is 0 . A very small discrepancy $(\sim 1.4 \%$ reduction) is obtained from the fabricated wax mold, which 
(a)

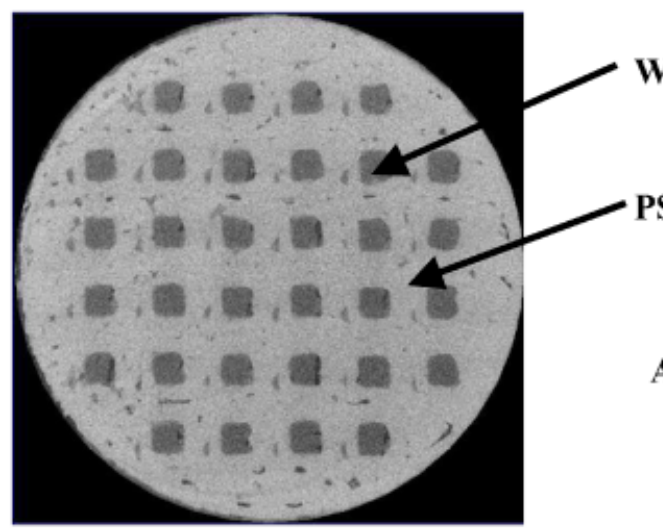

(b)

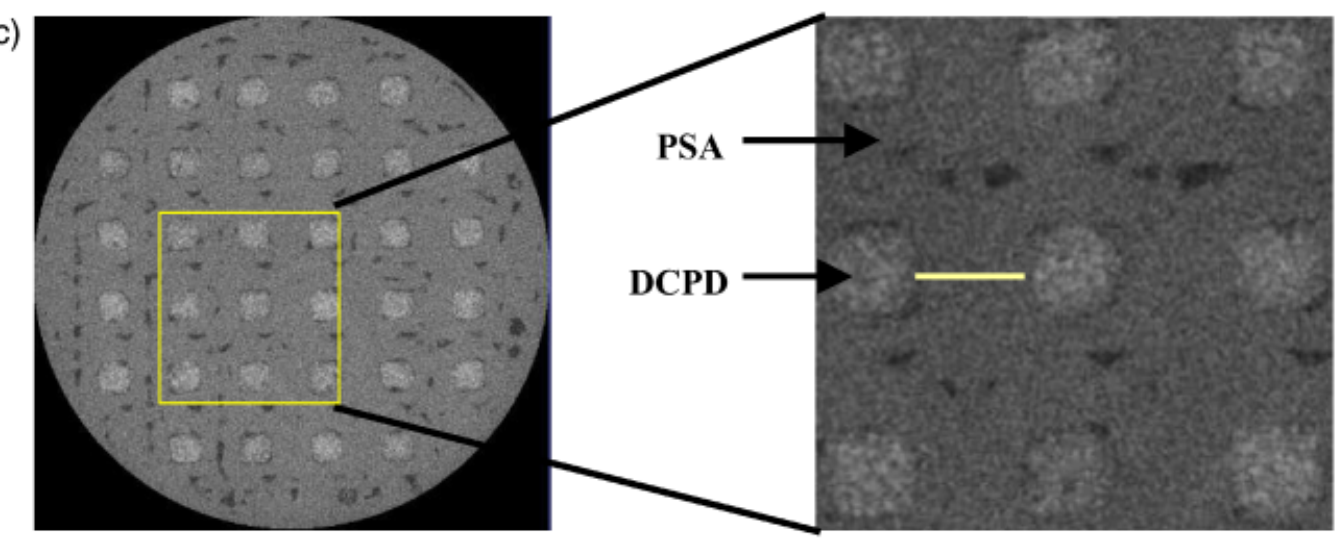

(d)

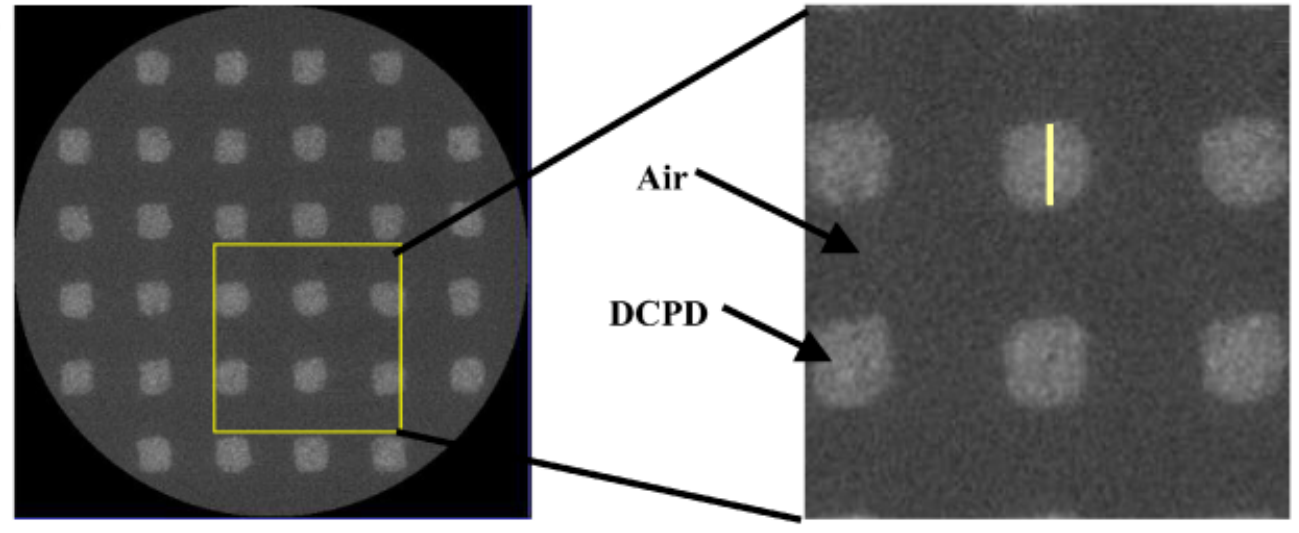

Fig. 3. Reconstructed micro-computed tomography images for the interior measurements: (a) Step 2-the wax mold, (b) Step 3 - the dewaxed mold; the inverted image is illustrated for better contrast, (c) Step 4 - the cast mold (left) and extended view of strut-size measurement (right), and (d) Step 5the dicalcium phosphate dihydrate (DCPD) scaffold (left) and pore-size measurement (right).

indicated a good performance of the fabrication method. This small change in the volume fraction may have to do with the configuration file for the scaffold layout on the ModelMaker II.

Table I. Physical Dimensions of Wax Molds, Dewaxed Molds, Cast Ceramic Molds, and DCPD Cement Scaffolds, $n=6$

\begin{tabular}{llcc}
\hline \multicolumn{1}{c}{ Fabrication process } & $\begin{array}{l}\text { Strut/channel } \\
\text { materials for } \\
\mu-C T \text { images }\end{array}$ & $\begin{array}{c}\text { Strut } \\
\text { size }(\mu \mathrm{m})\end{array}$ & $\begin{array}{c}\text { Channel } \\
\text { size }(\mu \mathrm{m})\end{array}$ \\
\hline Step 1: CAD design & NA & 550 & 650 \\
Step 2: Wax mold & Wax/PSA & $550 \pm 10$ & $650 \pm 10$ \\
Step 3: Dewaxed mold & Air/PSA & $540 \pm 10$ & $630 \pm 20$ \\
Step 4: Cast mold & DCPD/PSA & $540 \pm 20$ & $645 \pm 10$ \\
Step 5: DCPD scaffold & DCPD/air & $500 \pm 20$ & $690 \pm 25$ \\
\hline
\end{tabular}

DCPD, dicalcium phosphate dihydrate; $\mu$-CT, micro-computed tomography; PSA, polysulphonamide; NA, not applicable.
With a layer thickness of $0.0508 \mathrm{~mm}$, the configuration ID 471 - each layer is composed of two walls of Protosupport ${ }^{\mathrm{TM}}$ and three walls of Protobuild ${ }^{\mathrm{TM}}$, and the perimeter support over the entire part - was suitable for a wide variety of parts and was selected for this study. Although the finished mold appeared very solid externally, the inner layers of the wax mold were less dense because they were temporary supporting structures. The "smiley faces" and several defects (voids) on the reconstructed $\mu$-CT images (Fig. 3) were illustrated as a result of the internally "less dense" layers. The dewaxed molds showed a slight increase in the volume reduction caused by the dewaxing process. As mentioned in the experimental procedure, the wax was removed by melting in the oven at $80^{\circ}-85^{\circ} \mathrm{C}$ for $45 \mathrm{~min}$ to $1 \mathrm{~h}$. Note that the wax and the PSA melt between $50^{\circ}-75^{\circ} \mathrm{C}$ and $95^{\circ}-107^{\circ} \mathrm{C}$, respectively. Residual wax was further removed by washing in warm Bioact for $30 \mathrm{~min}$ and air drying. It is possible that the melted wax could induce PSA to melt especially at the interface, causing further reduction of the solid volume fraction. In this study, two types of calcium phosphate bioceramic scaffold 


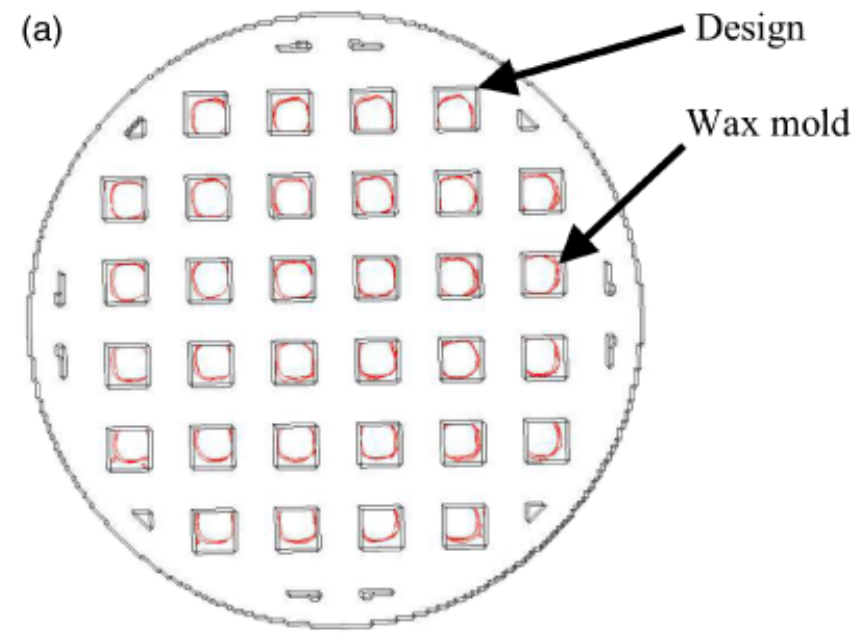

(b)

\section{Wax mold-DCPD}

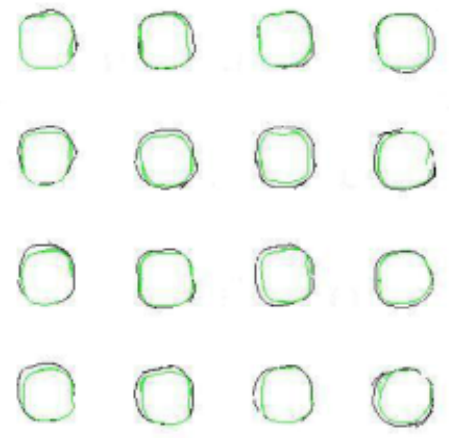

Fig. 4. Three-dimensional (3D) registration images of (a) Step 1-the computer-generated design channel size on the 3D isosurface micro-computed tomography image of Step 2-the wax mold, and (b) Step 2-wax mold on the isosurface of Step 5-the dicalcium phosphate dihydrate (DCPD) scaffold on the $x y$ plane. The image registration is performed using SolidView/Pro 2003.1.

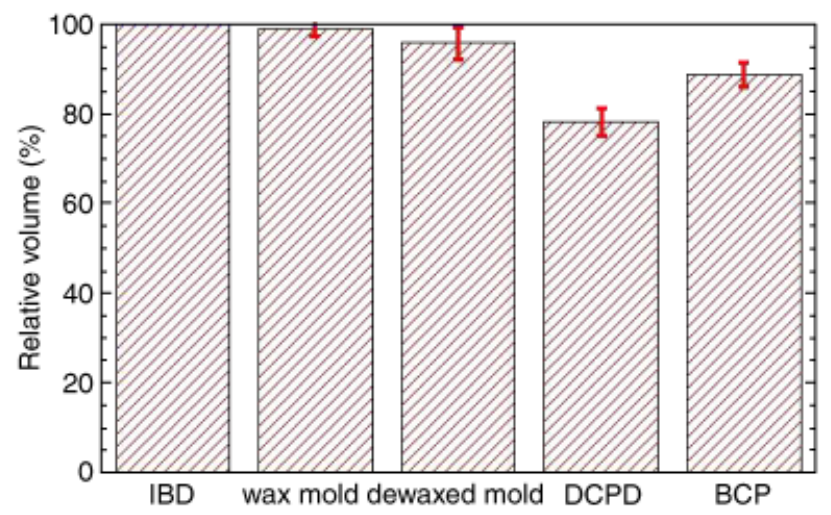

Fig. 5. Change in the relative volume fraction of solid. The reduction in the volume of dicalcium phosphate dihydrate (DCPD) scaffolds $(n=3)$ and biphasic calcium phosphate $(\mathrm{BCP})$ scaffolds $(n=3)$ corresponds to the internal pores and the shrinkage, respectively.

materials were represented: biphasic calcium phosphate $(\mathrm{BCP})$ and DCPD cement. For cast BCP, the PSA molds were subject to a burnout cycle that removed the organic binders and the mold. Using the same design, the size of the BCP model was 1.37 times larger than the DCPD model to account for the shrinkage of the $\mathrm{BCP}$ after sintering. For the DCPD cement scaffold, the PSA mold was removed by $70 \%$ ethanol and air dried. The presence of the internal pores causes a higher reduction in solid volume fraction on the DCPD scaffold than in the BCP scaffold. Note that by applying the ISFF technique with conventional casting methods, complex geometry and well-controlled internal architecture can be achieved. Several casting processes have been demonstrated for a combination of global and local pores ${ }^{7}$; therefore, with scaffolds made of other materials, the choice of solvent for mold dissolution will depend on the cast and mold materials. For this study, mold removal by solvent dissolution was the only possible method for non-sintered DCPD cement scaffolds.

The results obtained by the nondestructive approach allow us to examine the scaffold artifacts internally. Figure 6 shows the image of the $x z$ plane of one missing strut of the cast ceramic in the dewaxed mold, and two more struts were missing on the same plane after the mold removal. Any missing strut from a casting error may induce its nearest neighbor to break or erode during the mold dissolution, hence causing an increase in the number of missing struts in the finished scaffolds. If there is no missing strut during the casting, the possibility of obtaining a perfect scaffold is very high. Table II demonstrates the percentage of good struts formed over the entire scaffold, which is relatively high $(95 \%$ or more). These results indicate that the fabrication of ceramic scaffolds using indirect casting tools
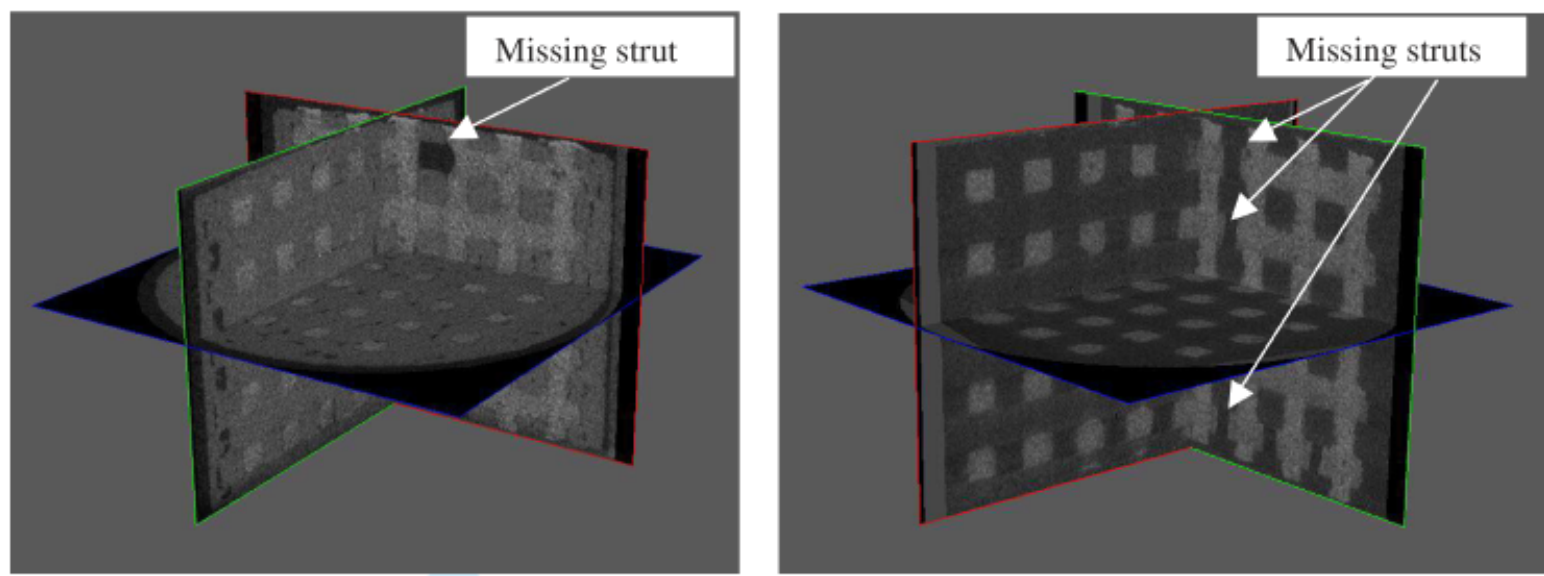

Fig. 6. Micro-computed tomography images show the missing struts of the cast mold (left) and of the dicalcium phosphate dihydrate (DCPD) scaffold after mold removal (right). 
Table II. Formation of Good Strut of the DCPD Scaffolds; the Volume of Interest was the Total Volume of the Scaffolds and the Number of Missing Struts of the Cast Cement Mold and the DCPD Scaffolds from the $\mu$-CT Images; the Region of Interest was the Middle Layer in the $X Z$ and $Y Z$ Planes for all Scaffolds

\begin{tabular}{lccc}
\hline & \multirow{2}{*}{$\begin{array}{c}\text { Percentage of good } \\
\text { Scaffold }\end{array}$} & \multicolumn{2}{c}{ Number of missing struts } \\
\cline { 3 - 4 } struts formation (per scaffold) & Cast mold & Scaffold \\
\hline Scaffold 1 & 98 & 1 & 2 \\
Scaffold 2 & 100 & None & None \\
Scaffold 3 & 98 & 1 & 4 \\
Scaffold 4 & 96 & 3 & 7 \\
\hline
\end{tabular}

DCPD, dicalcium phosphate dihydrate; $\mu$-CT, micro-computed tomography.

offers great potential for the production of complex 3D scaffolds with micro-interconnected networks. Note that precise structural control beyond random microporosity with a completely interconnected pore network has not been achieved by conventional processing techniques. ${ }^{19-22}$ Based on these results from $\mu$ $\mathrm{CT}$, we can conclude that the indirect scaffold fabrication using $3 \mathrm{D}$ printing via the casting technique shows a high potential to produce accurately the microstructure scaffolds with high-oriented pore size and shape internally.

\section{Conclusion}

We have demonstrated the application of $\mu$-CT to visualize and thoroughly characterize the internal architecture of manufactured scaffolds from the computer-aided design and rapid prototyping technologies and assess their porosity. Complex molds were designed and fabricated in a combination of wax and thermoplastic materials layer by layer using a commercial $3 \mathrm{D}$ printing system. The resolution of the $3 \mathrm{D}$ printer is limited by the nozzle size and the degree of control allowed over the position controller that defines the print-head movement. This method is shown to be accurate with $50 \mu \mathrm{m}$ or about $10 \%$ for nominally $650 \mu \mathrm{m}$ channels with $550 \mu \mathrm{m}$ struts. The struts in the DCPD scaffolds are about $50 \mu \mathrm{m}$ smaller than the design, with channels of about $40 \mu \mathrm{m}$ less than the design (Table II). $\mu$-CT can reveal the presence of the casting defect in the scaffold interior, results in missing struts. Casting methods and mold removal are subject to the materials of choice. Taken together, we demonstrated that the ISFF technique along with casting methods provide a greater degree of versatility and detail to scaffold design. These molds can meet specific tissue-engineering requirements, including the need for mechanical integrity. The representative DCPD cement scaffold was successfully fabricated and thoroughly characterized internally.

\section{Acknowledgment}

The authors would like to thank A. Diggs for supplying the BCP samples. This paper was presented in part at the 107th Annual Meeting of the American Ceramic
Society, Baltimore, MD, April 11, 2005 (Focused Symposium of: S-11-Bioceramics: Materials and Applications, Session: Calcium Phosphate Ceramics).

\section{References}

${ }^{1}$ C. P. A. T. Klein, P. Patka, and W. den Hollander, "Macroporous Calcium Phosphate Bioceramics in Dog Femora: A Histological Study of Interface and Biodegradation," Biomaterials, 10, 59-62 (1989).

${ }^{2}$ D. M. Liu, "Fabrication of Hydroxyapatite Ceramic with Controlled Porosity," J. Mater. Sci.:Mater. Med., 8, 227-32 (1997).

${ }^{3}$ D. M. Liu, "Influence of Porosity and Pore Size on the Compressive Strength of Porous Hydroxyapatite Ceramic," Ceram. Int., 23, 135-9 (1997).

${ }^{4}$ T. M. G. Chu, D. G. Orton, S. J. Hollister, S. E. Feinberg, and J. W. Halloran, "Mechanical and In Vivo Performance of Hydroxyapatite Implants with Controlled Architectures," Biomaterials, 23, 1283-93 (2002).

${ }^{5}$ S. J. Hollister, R. A. Levy, T. M. Chu, J. W. Halloran, and S. E. Feinberg, “An Image-Based Approach for Designing and Manufacturing Craniofacial Scaffolds," Int. J. Oral Maxillofac. Surg., 29, 67-71 (2002).

${ }^{6}$ S. J. Hollister, R. D. Maddox, and J. M. Taboas, "Optimal Design and Fabrication of Scaffolds to Mimic Tissue Properties and Satisfy Biological Constraints," Biomaterials, 23, 4095-103 (2002).

7J. M. Taboas, R. D. Maddox, P. H. Krebsbach, and S. J. Hollister, "Indirect Solid Freeform Fabrication of Local and Global Porous, Biomimetic and Composite 3D Polymer-Ceramic Scaffolds," Biomaterials, 24, 181-94 (2003).

${ }^{8}$ T. M. G. Chu, J. W. Halloran, S. J. Hollister, and S. E. Feinberg, "Hydroxyapatite Implants with Designed Internal Architecture," J. Mater. Sci.: Mater. Med., 12, 471-8 (2001)

${ }^{9}$ C. J. Reilly, W. J. Chappell, J. W. Halloran, and L. P. B. Katehi, "HighFrequency Electromagnetic Bandgap Structures via Indirect Solid Freeform Fabrication," J. Am. Ceram. Soc., 87 [8] 1446-53 (2004).

${ }^{10}$ L. Jongpaiboonkit, T. M. G. Chu, and J. W. Halloran, "Characterization and Rheology Behavior of DCPD Cement Slips for Casting Complex Scaffold"; Society for Biomaterials 2003 Annual Meeting, Reno, NV.

${ }^{11}$ L. Jongpaiboonkit, C. Y. Lin, P. H. Krebsbach, S. J. Hollister, and J. W. Halloran, "Mechanical Behavior of 3D Calcium Phosphate Cement Scaffolds Fabricated by Indirect Solid Freeform Fabrication In Vivo," Bioceramics, 18, 957 60 (2005).

${ }^{12}$ C. P. A. T. Klein, A. A. Driessen, K. de Groot, and A. van den Hooff, "Biodegradation Behavior of Various Calcium Phosphate Materials in Bone Tissue," J. Biomed. Mater. Res., 17, 769-84 (1983).

${ }^{13}$ A. Uchida, E. Kurisaki, and K. Ono, "The Use of Bioactive Ceramics for Bone Tumor Surgery"; pp. 345-4 in Handbook of Bioactive Ceramicss, Vol. 11, Calcium Phosphate and Hydroxyapatite Ceramics, Edited by T. Yamamuro, L. L. Hench, and J. Wilsons. CRC Press, Boca Raton, FL, 1990.

${ }^{14}$ W. E. Brown and L. C. Chow, "A New Calcium Phosphate Setting Cement," J. Dent. Res., 62, 672 (1983).

${ }^{15}$ A. A. Mirtchi, J. Lamaitre, and E. Munting, "Calcium Phosphate Cements: Action of Setting Regulators on the Properties of the $\beta$-Tricalcium PhosphateMonocalcium Phosphate Cements," Biomaterials, 10, 634-8 (1989).

${ }^{16}$ S. J. Hollister, "Porous Scaffold Design for Tissue Engineering," Nat. Mater., 4, 518-24 (2005).

${ }^{17}$ C. Y. Lin, N. Kikuchi, and S. J. Hollister, "A Novel Method for Biomaterial Scaffold Internal Architecture Design to Match Bone Elastic Properties with Desired Porosity," J. Biomech., 37, 623-36 (2004).

${ }^{18}$ E. Sachlos, N. Reis, C. Ainsley, B. Derby, and J. T. Czernuszka, "Novel Collagen Scaffolds with Predefined Internal Morphology Made by Solid Freeform Fabrication," Biomaterials, 24, 1487-97 (2003).

${ }^{19}$ M. J. Cima, E. Sachs, L. G. Cima, J. Yoo, S. Khanuja, S. W. Borland, B. Wu, and R. A. Giordano, "Computer-Derived Microstructures by 3D Printing: Bioand Structural Materials"; pp. 181-90 in Solid Freeform Fabrication Symposium 1994 Proceedings, Edited by H. L. Marcus, J. J. Beaman, J. W. Barlow, D. L. Bourell, and R. H. Crawford. The University of Texas at Austin, Austin, TX, 1994.

${ }^{20}$ S. L. Ishaug-Riley, G. M. Crane-Kruger, M. J. Yaszemski, and A. G. Mikos, "Three-Dimensional Culture of Rat Calvarial Osteoblasts in Porous Biodegradable Polymers," Biomaterials, 19, 1405-12 (1998).

${ }^{21}$ P. X. Ma and R. J. Zhang, "Synthetic Nano-Scale Fibrous Extracellular Matrix," J. Biomed. Mater. Res., 46, 60-72 (1999).

${ }^{22}$ W. L. Murphy, R. G. Dennis, J.L Kileny, and D. J. Mooney, "Salt Fusion: An Approach to Improve Pore Interconnectivity Within Tissue Engineering Scaffolds," Tissue Eng., 8, 43-52 (2002). 